\title{
An imprinted IMAGe: insights into growth regulation through genomic analysis of a rare disease
}

\author{
Renuka P Dias',2 and Eamonn R Maher, ${ }^{1,3}$
}

\begin{abstract}
Missense mutations in the imprinted gene that encodes cyclin-dependent kinase inhibitor 1C (CDKN1C, also called p57Kip2) result in a rare disorder associated with prenatal growth retardation (IMAGe syndrome). Lossof-function mutations in CDKN1C have been previously described in the congenital overgrowth syndrome Beckwith-Wiedemann syndrome and some cancers. In contrast, a recent study by Arboleda et al. proposes that the CDKN1C mutations associated with IMAGe syndrome have a gain-of-function effect. These findings highlight how rare genetic disorders can provide important insights into the regulation of critical processes such as regulation of cell growth.
\end{abstract}

There is little relationship between the incidence of a disease and the mechanistic insights that can be derived from elucidating its molecular mechanisms. Most rare diseases are inherited, and advances in genomic technologies are rapidly increasing our knowledge of their genetic basis and the pathways that regulate key cellular processes. The exquisite phenotyping of human diseases is enabling intriguing genotype-phenotype relationships to be uncovered, as exemplified by a recent report by Arboleda et al. in Nature Genetics [1] on the role of cyclin-dependent kinase inhibitor 1C (CDKN1C, p57Kip2) mutations in the growth retardation syndrome IMAGe and the overgrowth syndrome Beckwith-Wiedemann syndrome (BWS) [2].

IMAGe syndrome (Mendelian Inheritance in Man (MIM) ID 300290) is a very rare inherited disorder characterized by intra-uterine growth restriction,

\footnotetext{
*Correspondence: e.r.maher@bham.ac.uk

'Centre for Rare Diseases and Personalized Medicine, School of Clinical and

Experimental Medicine, College of Medical and Dental Sciences, University of

Birmingham, Edgbaston, Birmingham B15 2TT, UK

Full list of author information is available at the end of the article
}

abnormal bones (metaphyseal dysplasia), congenital adrenal gland insufficiency (adrenal hypoplasia congenita) and genital anomalies (Table 1) [3]. Arboleda et al. [1] studied a very large family who have IMAGe syndrome and using array-based SNP genotyping and targeted second generation resequencing methods, they identified a region on chromosome 11p that was shared by all affected individuals and detected a heterozygous CDKN1C missense mutation that all affected individuals had inherited from their mother. Further analysis of unrelated patients with IMAGe syndrome revealed additional $C D K N 1 C$ missense mutations and, remarkably, all five distinct missense mutations that were characterized occurred in the carboxy-terminal region of CDKN1C, within a cluster of six amino acids in the proliferating cell nuclear antigen (PCNA)-binding domain. CDKN1C is a maternally expressed imprinted gene that maps within the 11p15.5 imprinted gene cluster and all individuals affected by IMAGe syndrome had inherited the mutation on the maternal allele.

BWS is characterized by pre- and postnatal overgrowth, macroglossia (large tongue), anterior abdominal wall defects and susceptibility to embryonal tumors (most commonly Wilms tumor). Previously, maternally inherited CDKN1C mutations have been demonstrated to cause up to $50 \%$ of familial and $5 \%$ of sporadic cases of BWS [2]. Furthermore, about $50 \%$ of sporadic BWS cases have epigenetic silencing of maternal CDKN1C allele expression, secondary to epimutations at an 11p15.5 imprinting control center (IC2/KvDMR1) [2,4]. In contrast to the restricted type and location of CDKN1C mutations in IMAGe syndrome, those in BWS-associated CDKN1C are most often truncating mutations distributed throughout the gene, or missense mutations in the aminoterminal CDK inhibitor domain, suggesting that the mutations causing IMAGe syndrome and BWS have different functional effects [1].

\section{CDKN1C mutations and function}

Arboleda et al. [1] found that whereas BWS-associated CDKN1C mutations caused loss of cell cycle inhibition, 
Table 1. Human inherited disorders associated with abnormal CDKN1C function

\begin{tabular}{|c|c|c|c|}
\hline & $\begin{array}{l}\text { IMAGe syndrome } \\
\text { (MIM 300290) }\end{array}$ & $\begin{array}{l}\text { Silver-Russell syndrome } \\
\text { (MIM 180860) }\end{array}$ & $\begin{array}{l}\text { Beckwith-Wiedemann syndrome } \\
\text { (MIM 130650) }\end{array}$ \\
\hline Main clinical features & $\begin{array}{l}\text { Intra-uterine growth restriction, } \\
\text { metaphyseal dysplasia, adrenal } \\
\text { hypoplasia congenita, genital anomalies }\end{array}$ & $\begin{array}{l}\text { Intra-uterine growth restriction, relative } \\
\text { macrocephaly, triangular shaped face, } \\
\text { hemihypoplasia, }\end{array}$ & $\begin{array}{l}\text { Pre and/or postnatal overgrowth, } \\
\text { macroglossia, anterior abdominal } \\
\text { wall defects, neonatal hypoglycemia, } \\
\text { hemihypertrophy, predisposition to } \\
\text { embryonal tumors }\end{array}$ \\
\hline CDKN1Cabnormalities & $\begin{array}{l}\text { Missense mutations in the PCNA-binding } \\
\text { domain (maternal allele) }\end{array}$ & $\begin{array}{l}\text { Microduplication of CDKN1C (maternal } \\
\text { allele) }\end{array}$ & $\begin{array}{l}\text { Loss-of-function CDKN1C mutations } \\
\text { (maternal allele), epigenetic silencing by } \\
\text { epimutations (loss of methylation) and } \\
\text { mutations of the KvDMR1 imprinting } \\
\text { control center (IC2) (maternal allele) }\end{array}$ \\
\hline Other genetic causes & - & $\begin{array}{l}\text { Epimutations (loss of methylation) of } \\
\text { H19/IGF2 intergenic imprinting control } \\
\text { center (IC1; paternal allele), maternal } \\
\text { disomy chromosome } 7\end{array}$ & $\begin{array}{l}\text { Epimutations (gain of methylation) and } \\
\text { mutations H19/IGF2 intergenic imprinting } \\
\text { control center (IC1) (maternal allele) }\end{array}$ \\
\hline
\end{tabular}

IMAGe-associated mutations had no effect on the ability of CDKN1C to inhibit the G0-to-G1 cell cycle transition. Evidence for a gain-of-function effect of IMAGeassociated $C D K N 1 C$ mutations was detected in an in vivo Drosophila melanogaster assay, in which expression of the mutant but not the wild-type human protein reduced eye and wing size. PCNA has crucial roles in DNA replication and cell cycle control (by facilitating ubiquitination and degradation of cell-cycle proteins) [5]. Arboleda et al. [1] found that IMAGe-associated CDKN1C mutations inhibited binding to PCNA and monoubiquitination of CDKN1C. Impaired ubiquitination of CDKN1C might have a number of functional consequences, and further studies are required to elucidate precisely how IMAGeassociated CDKN1C mutations produce a gain-of-function effect (including their effect on protein stability). Although ubiquitin status can be an important determinant of protein stability, Arboleda et al. [1] point out that proteasomal degradation is typically induced by polyubiquitination, so alterations in monoubiquitination status probably influence function by an alternative mechanism. Discovering of the exact pathogenic mechanisms of IMAGe CDKN1C mutations could provide a basis for developing new therapeutic strategies and targets, both for IMAGe and for other diseases associated with CDKN1C dysregulation.

Clinical phenotypes of CDKN1C spectrum disorders Genomic imprinting refers to the expression of genes from only one of the two chromosome homologs depending on whether the copy is maternally or paternally inherited. Imprinted genes account for less than $1 \%$ of all human genes, but those identified so far seem to be preferentially implicated in the regulation of growth and development. Furthermore, in general, paternally expressed genes (such as that encoding insulin-like growth factor 2 (IGF2)) promote growth, whereas maternally expressed imprinted genes (such as CDKN1C) suppress growth. These observations have been used to support the kinship (or parental conflict) theory for the evolution of parental imprinting in which it is suggested that paternally expressed imprinted genes will favor fetal growth over maternal health, whereas maternally expressed genes act to limit fetal growth so as to preserve maternal fitness and allow further progeny [6]. IMAGe syndrome and BWS are both associated with abnormal prenatal growth (under- and overgrowth, respectively). In addition, Silver-Russell syndrome (MIM 180860; Table 1), which is characterized by pre- and postnatal growth failure, is most commonly associated with epimutations leading to loss of IGF2 expression, but has occasionally been associated with a maternal chromosome duplication that includes CDKN1C $[7,8]$. Interestingly, CDKN1C is highly expressed in the developing adrenal gland and different mutation types present different adrenal phenotypes: IMAGe syndrome is associated with congenital adrenal hypoplasia, whereas BWS may be associated with adrenocortical tumors, and sporadic adrenocortical tumors can show CDKN1C inactivation $[2,3,9]$.

\section{Conclusions}

The application of high-throughput genomic analysis techniques enabled Arboleda et al. [1] to define the molecular basis of IMAGe syndrome. This breakthrough will facilitate diagnosis of the disorder and better assessment of genetic risk in affected families. In addition to illuminating $C D K N 1 C$ growth regulatory mechanisms, Arboleda et al. [1] provide a further example of how the mutational diversity of inherited diseases in humans can provide a rich source of insights into basic biology and pathobiology - as extolled by William Harvey more than 300 years ago: 'Nature is nowhere accustomed more openly to display her secret mysteries than in cases where 
she shows traces of her workings apart from the beaten path; nor is there any better way to advance the proper practice of medicine than to give our minds to the discovery of the usual law of Nature by careful investigation of cases of rare forms of disease' [10].

\section{Abbreviations}

BWS, Beckwith-Wiedemann syndrome; CDKN1C, cyclin-dependent kinase inhibitor $1 C$; IGF2, insulin-like growth factor 2; MIM, Mendelian Inheritance in Man; PCNA, proliferating cell nuclear antigen.

\section{Competing interests}

The authors declare that they have no competing interests.

\section{Authors' contributions}

RPD and ERM co-wrote the article.

\section{Author details}

${ }^{1}$ Centre for Rare Diseases and Personalized Medicine, School of Clinical and Experimental Medicine, College of Medical and Dental Sciences, University of Birmingham, Edgbaston, Birmingham B15 2TT, UK. 'Department of Endocrinology, Birmingham Children's Hospital, Steelhouse Lane, Birmingham B4 6NH, UK. 'West Midlands Regional Genetics Service, Birmingham Women's Hospital, Edgbaston, Birmingham B15 2TG, UK.

Published: 30 July 2012

\section{References}

1. Arboleda VA, Lee H, Parnaik R, Fleming A, Banerjee A, Ferraz-de-Souza B, Délot EC, Rodriguez-Fernandez IA, Braslavsky D, Bergadá I, Dell'angelica EC, Nelson SF, Martinez-Agosto JA, Achermann JC, Vilain E: Mutations in the PCNAbinding domain of CDKN1C cause IMAGe syndrome. Nat Genet 2012, 44:788-792.
2. Lim DH, Maher ER: Genomic imprinting syndromes and cancer. Adv Genet 2010, 70:145-175.

3. Vilain E, Le Merrer M, Lecointre C, Desangles F, Kay MA, Maroteaux P, McCabe ER: IMAGe, a new clinical association of intrauterine growth retardation, metaphyseal dysplasia, adrenal hypoplasia congenita, and genital anomalies. J Clin Endocrinol Metab 1999, 84:4335-4340.

4. Diaz-Meyer N, Day CD, Khatod K, Maher ER, Cooper W, Reik W, Junien C, Graham G, Algar E, Der Kaloustian VM, Higgins MJ: Silencing of CDKN1C (p57KIP2) is associated with hypomethylation at KvDMR1 in BeckwithWiedemann syndrome. J Med Genet 2003, 40:797-801.

5. Havens CG, Walter JC: Mechanism of CRL4(Cdt2), a PCNA-dependent E3 ubiquitin ligase. Genes Dev 2011, 25:1568-1582.

6. Wilkins JF, Haig D: What good is genomic imprinting: the function of parent-specific gene expression. Nat Rev Genet 2003, 4:359-368.

7. Bonaldi A, Mazzeu JF, Costa SS, Honjo RS, Bertola DR, Albano LM, Furquim IM, Kim CA, Vianna-Morgante AM: Microduplication of the ICR2 domain at chromosome 11 p15 and familial Silver-Russell syndrome. Am J Med Genet A 2011, 155A:2479-2483.

8. Schönherr N, Meyer E, Roos A, Schmidt A, Wollmann HA, Eggermann T: The centromeric $11 \mathrm{p} 15$ imprinting centre is also involved in Silver-Russell syndrome. J Med Genet 2007, 44:59-63.

9. Bourcigaux N, Gaston V, Logié A, Bertagna X, Le Bouc Y, Gicquel C: High expression of cyclin E and G1 CDK and loss of function of p57KIP2 are involved in proliferation of malignant sporadic adrenocortical tumors. J Clin Endocrinol Metab 2000, 85:322-330.

10. Harvey W: Letters. In The Works of William Harvey. Edited by Alfred Fishman Philadelphia: University of Pennsylvania Press; 1989:616-617.

doi:10.1186/gm361

Cite this article as: Dias RP, Maher ER: An imprinted IMAGe: insights into growth regulation through genomic analysis of a rare disease. Genome Medicine 2012, 4:60 